\title{
R Resercrch Suare \\ The Effect of Posterior Draw During Graft Fixation of Anterior Cruciate Ligament Reconstruction
}

\section{Sung-Sahn Lee ( $\nabla$ sungsahnlee@gmail.com )}

Inje University Ilsan Paik Hospital https://orcid.org/0000-0001-7728-0061

\section{Jin-Ho Cho}

Inje University Ilsan Paik Hospital

Jae Won Heo

Inje University Ilsan Paik Hospital

\section{Research Article}

Keywords: Graft, anterior cruciate ligament reconstruction, draw

Posted Date: January 24th, 2022

DOI: https://doi.org/10.21203/rs.3.rs-1138357/v1

License: (c) (i) This work is licensed under a Creative Commons Attribution 4.0 International License.

Read Full License 


\section{Abstract}

Background: We think reduction forced toward the posterior side during graft fixation could be helpful in reducing the side-to-side difference (STSD) after ACL reconstruction. The purpose of our study was to compare the clinical and radiological outcomes of graft fixation with or without a posterior draw during anterior cruciate ligament $(\mathrm{ACL})$ reconstruction surgery.

Methods: Among 110 patients who underwent primary arthroscopic ACL reconstruction between January 2017 and August 2020, 76 patients underwent surgery without a posterior draw (non-draw group) and 34 patients underwent ACL reconstruction with posterior draw (draw group). Results of the Lachman test, pivot-shift test, Western Ontario and McMaster Universities Osteoarthritis (WOMAC) index, Lysholm score, International Knee Documentation Committee (IKDC) subjective score, and STSD on stress radiography were compared between the two groups.

Results: The postoperative WOMAC index, Lysholm score, and IKDC subjective score were similar with the draw group. Postoperative STSD (non-draw group vs draw group, $2.4 \pm 2.2$ vs $2.0 \pm 2.2, P=0.319$ ) and change of STSD (preoperative STSD vs postoperative STSD, $3.5 \pm 3.5$ vs $4.3 \pm 4.4, P=0.295$ ) were not superior in the draw group.

Conclusion: Graft fixation with a posterior draw was not helpful in enhancing postoperative stability during ACL reconstruction. Postoperative clinical outcomes were also not superior in the draw group.

Study design: Level III, retrospective comparative study.

\section{Introduction}

Anterior cruciate ligament (ACL) tears are a common orthopedic injury that most frequently affect young and active patients.[24] For those interested in returning to high-level athletic competitions, ACL reconstruction is widely performed with satisfactory results.[23] The anatomical ACL reconstruction allowed us to obtain accurate restoration of native ACL positioning and biomechanical characteristics.[1; 8; 17] However, some patients had poor outcomes with residual instability after surgery. $[6 ; 11 ; 21]$ Numerous factors were associated with stability after ACL reconstruction, including graft selection, tunnel position, and extra-articular structure injury (ex. anterolateral ligament). $[12 ; 14 ; 20]$ Among them optimal fixation is one of the important factors for successful reconstruction. [7; 13]

Optimal fixation of soft tissue grafts in ACL reconstruction, including graft selection, optimal tension, fixative method (aperture fixation vs. suspensory fixation), and knee flexion angle during fixation remains a controversial topic. [9; 13; 26] Almekinders et al.[3] had first reported the concept of static anterior tibial subluxation after ACL injury, and the abnormal static relationship between the femur and tibia with the knee in extension. Subsequently, several studies investigated the static anterior tibial subluxation after ACL injury. $[2 ; 4 ; 20 ; 25]$ Therefore, we think reduction forced toward the posterior side during graft fixation could be helpful in reducing the side-to-side difference (STSD) after ACL reconstruction.[22] (Fig. 1) 
This study aimed to compare the clinical and radiological outcomes of graft fixation with or without a posterior draw. We hypothesized that graft fixation with a posterior draw would reduce postoperative STSD following ACL reconstruction.

\section{Materials And Methods}

\section{Patients}

This was a retrospective study of enrolled patients who underwent primary arthroscopic ACL reconstruction between January 2017 and August 2020 at our institution. The inclusion criteria were: 1) ACL total rupture, diagnosed by magnetic resonance imaging and arthroscopic examination, 2) performed arthroscopic ACL reconstruction, and 3) a follow-up period of more than 12 months. The exclusion criteria were as follows: 1) osteoarthritic changes in the injured knee, 2) multiple-ligament reconstruction, or 3) contralateral knee ACL reconstruction history. A total of 110 patients was enrolled in this study. Patients were divided into two groups: those who underwent graft fixation with posterior draw (draw group) and those who underwent graft fixation without posterior draw (non-draw group). Among the 110 patients, 76 patients were in the non-draw group and 34 in the draw group. The study protocol was approved by our institutional review board (ISPAIK 2021-09-019)

\section{Surgical Procedures}

The operations were performed by two fellowship-trained surgeons (J.H.C. and S.S.L.). One surgeon preferred the posterior draw of the tibia during graft fixation, while the other surgeon did not. The remaining surgical procedures were similar. All patients were able to select the graft type (autograft or allograft) after sufficient explanation. The hamstring tendon was harvested, and a 4-strand double-loop single-bundle graft was inserted in reconstruction with an autograft. If patients opted for an allograft, the allogenous tibialis anterior tendon was used for ACL reconstruction. A mixed graft was used when the diameter of the harvested autograft was too small for application.

Portal formation and arthroscopic examinations were performed in the standard manner. Combined meniscal tears were also evaluated. The femoral tunnel was formed using trans anteromedial portal method.[15] The center of the anatomical footprint was marked with a microfracture awl (ConMed [Linvatec]), after removal of the ACL remnant tissue. A $2.4 \mathrm{~mm}$ guide pin was inserted with the knee fully flexed, then a $4.5 \mathrm{~mm}$ EndoButton drill (Smith \& Nephew) was used to drill through the far cortex. After measuring the femoral tunnel length, the femoral tunnel was formed using a cannulated reamer. To form the tibial tunnel, a guide wire was inserted from the medial tibial cortex to the footprint of the ACL using a Pinn-ACL guide (Linvatec), and the tibial tunnel was created using a cannulated reamer. The EndoButton drill (Smith \& Nephew) was used for femoral side graft fixation. After the graft was passed, the position of the EndoButton was checked using C-arm fluoroscopy. Tensioner was routinely used to check the initial tension (target: $25 \mathrm{~N}$ ). Hybrid fixation, which combined intra-tunnel aperture and extracortical suspensory fixation, was used for tibial side fixation. $[7 ; 26]$ The posterior draw force was applied when 
tibial aperture fixation was performed in the draw group, and draw force was not applied in the non-draw group. (Fig. 2)

Partial weight-bearing walking with crutches was allowed for the initial 4 weeks after reconstruction surgery, and full weight-bearing walking was permitted at 6 weeks. Range of motion (ROM) exercise was started 2 days after surgery and reached $120^{\circ}$ of knee flexion by 4 weeks. Straight-leg raises, quadriceps sets, and ankle pump exercises were started on the first postoperative day, closed kinetic chain exercise was initiated 2 weeks postoperatively, and return to sports was allowed after 9 months, depending on the patient's condition.

\section{Clinical and radiographic evaluation}

Demographic data, including age, sex, body mass index (BMI), and time from injury to reconstruction surgery, were obtained. Pre- and postoperative clinical outcomes were gathered using the following evaluations: Lachman test, pivot-shift test, Western Ontario and McMaster Universities Osteoarthritis (WOMAC) index,[10] Lysholm score[18], and International Knee Documentation Committee (IKDC) subjective score.[5] The Lachman test was graded as 0, 1 (<5 mm), 2 (5 to $10 \mathrm{~mm})$, or 3 (>10 mm) compared to the contralateral knee, and the Pivot-shift test was graded as 0 (absent), 1 (glide), 2 (clunk), or 3 (gross).[16]

Preoperative and postoperative telos stress radiography ( $15 \mathrm{~kg}$ on the tibia at $20^{\circ}$ of knee flexion) was evaluated to measure STSD. (Fig. 3) Anterior tibial translation was investigated from the radiographs by measuring the distance from the posterior margin of the tibial condyle to the femoral condyles. STSD was defined as the difference between the anterior tibial translations of both knees. The STSD was evaluated by two independent orthopedic surgeons (SHC and BHK) specializing in ACL reconstruction, who did not participate in the current study, to verify interobserver reliability. The intraobserver reliability was checked by having the observers repeat the same measurements 6 weeks later. Intra-class correlation coefficients (ICCs) were used for inter- and intra-observer reliability assessments.

Preoperative and postoperative outcomes were compared, and all outcomes were compared between the draw and non-draw groups.

\section{Statistical analysis}

The Shapiro-Wilk test was used to evaluate the normality of the distribution. A paired $t$-test was used to compare the preoperative and postoperative outcomes. To compare the demographic data and preoperative and postoperative outcomes between both groups, Student's $t$-test or the Mann-Whitney $U$ test was used for continuous variables, whereas the chi-squared test or Fisher's exact test was used for categorical variables. Statistical significance was set at $p<0.05$. All data was analyzed using SPSS version 27.0 (IBM Corp., Armonk, NY, USA). In our study, 76 and 34 knees were allocated to the non-draw and draw groups, respectively. It would be $99 \%$ power to detect a difference of at $1 \mathrm{~mm}$ in the mean STSD with a standard deviation of $1 \mathrm{~mm}$ in a previous study $(a=0.05) .[15]$ 


\section{Results}

The inter - and intra-observer ICC of the STSD showed good agreement with respect to the reliability of the radiographic measurement $(>0.80)$. The demographic data are presented in Table 1.

Table 1

Demographic data

\begin{tabular}{|ll|}
\hline No. of patients ultimately enrolled & $\mathbf{1 1 0}$ \\
\hline Male: Female & $92: 18$ \\
\hline Age, year & $31.3 \pm 11.9$ \\
\hline Height, $\mathrm{cm}$ & $171.9 \pm 7.1$ \\
\hline Weight, $\mathrm{kg}$ & $75.8 \pm 12.6$ \\
\hline Body mass index, $\mathrm{kg} / \mathrm{m}^{2}$ & $25.5 \pm 3.3$ \\
\hline Graft & autograft, hamstring tendon: 66 \\
& allograft, tibialis anterior tendon: 35 \\
& mixed: 9 \\
\hline Time from injury to surgery, weeks & $8.5 \pm 14.2$ \\
\hline
\end{tabular}

Postoperative Lachman test and pivot-shift test grades improved compared to the preoperative grades. Postoperative clinical outcomes, including the WOMAC index, Lysholm score, and IKDC subjective score, were greater than the preoperative values. Postoperative STSD was significantly less than preoperative STSD. $(2.3 \pm 2.2$ vs $6.0 \pm 3.4, P<0.001$, Table 2$)$

Table 2

Comparison of preoperative and postoperative outcomes

\begin{tabular}{|llll|}
\hline & Preoperative & Postoperative & Pvalue \\
\hline Lachman test (Grade 0 / 1 / / 3) & $1 / 8 / 33 / 68$ & $89 / 21 / 0 / 0$ & $<0.001$ \\
\hline Pivot-shift test (Grade 0 / 1 / / 3) & $3 / 20 / 50 / 37$ & $99 / 11 / 0 / 0$ & $<0.001$ \\
\hline WOMAC index & $36.8 \pm 23.9$ & $10.6 \pm 13.2$ & $<0.001$ \\
\hline Lysholm score & $54.8 \pm 23.9$ & $84.2 \pm 13.2$ & $<0.001$ \\
\hline IKDC subjective score & $49.1 \pm 15.2$ & $73.7 \pm 13.0$ & $<0.001$ \\
\hline STSD, mm & $6.0 \pm 3.4$ & $2.3 \pm 2.2$ & $<0.001$ \\
\hline $\begin{array}{l}\text { STSD, Side-to side difference; WOMAC, Western Ontario and McMaster Universities Osteoarthritis; } \\
\text { IKDC, International Knee Documentation Committee }\end{array}$ & & \\
\hline
\end{tabular}


Demographic data and preoperative outcomes were similar between both groups. The combined meniscal lesions were not statistically different. The postoperative WOMAC index, Lysholm score, and IKDC subjective score were not greater in the draw group. Postoperative STSD (non-draw group vs draw group, $2.4 \pm 2.2$ vs $2.0 \pm 2.2, P=0.319$ ) and change of STSD (preoperative STSD - postoperative STSD, $3.5 \pm 3.5$ vs $4.3 \pm 4.4, P=0.295$ ) were not superior in draw group. (Table 3 ) 
Table 3

Comparison outcomes between both groups

Non-draw group

Draw group

$P$ value

Demographic data

Number of patients

76

34

Age, year

$30.8 \pm 12.1$

$32.6 \pm 11.6$

0.472

Sex, M: F

64: 12

28: 6

0.787

$\mathrm{BMl}, \mathrm{kg} / \mathrm{m} 2$

$25.2 \pm 3.2$

$26.2 \pm 3.6$

0.167

Time from injury to surgery, weeks

$9.2 \pm 14.2$

$7.2 \pm 14.1$

0.5

Follow up period, months

$18.6 \pm 8.6$

$16.7 \pm 5.4$

0.228

Graft (Autograft / allograft / mixed)

$43 / 25 / 8$

$23 / 10 / 1$

0.407

Combined medial meniscal tear

Medial

$22(28.9 \%)$

$10(29.4 \%)$

0.96

Lateral

$27(35.5 \%)$

$9(26.5 \%)$

0.387

Preoperative data

Lachman test (Grade 0 / 1 / 2 / 3)

$0 / 7 / 20 / 49$

$1 / 1 / 13 / 19$

0.499

Pivot-shift test (Grade 0 / 1 / 2 / 3)

$3 / 17 / 31 / 25$

$0 / 3 / 19 / 12$

0.217

WOMAC index

$35.2 \pm 25.9$

$39.4 \pm 18.5$

0.396

Lysholm score

$55.9 \pm 24.5$

$52.4 \pm 21.8$

0.472

IKDC subjective score

$50.6 \pm 15.3$

$46.1 \pm 14.6$

0.154

STSD, $\mathrm{mm}$

$5.9 \pm 3.0$

$6.3 \pm 4.3$

0.68

Postoperative data

Lachman test (Grade 0 / 1 / 2 / 3)

Pivot-shift test (Grade 0 / 1 / 2 / 3)

WOMAC index

$60 / 16 / 0 / 0$

$29 / 5 / 0 / 0$

0.601

68 / 8 / 0 / 0

$31 / 3 / 0 / 0$

0.542

Lysholm score

$10.3 \pm 15.0$

$10.7 \pm 8.1$

0.892

IKDC subjective score

$83.5 \pm 15.3$

$86.1 \pm 6.5$

0.345

STSD, $\mathrm{mm}$

$74.3 \pm 12.4$

$72.9 \pm 14.3$

0.603

$2.4 \pm 2.2$

$2.0 \pm 2.2$

0.319

STSD, Side-to side difference; WOMAC, Western Ontario and McMaster Universities Osteoarthritis; IKDC, International Knee Documentation Committee 


\begin{tabular}{|c|c|c|c|}
\hline & Non-draw group & Draw group & $P$ value \\
\hline Change of STSD (pre to post), mm & $3.5 \pm 3.5$ & $4.3 \pm 4.4$ & 0.295 \\
\hline
\end{tabular}

\section{Discussion}

The principal finding of the current study is that the posterior draw force is not helpful in enhancing postoperative stability during $\mathrm{ACL}$ graft fixation. Moreover, the postoperative clinical outcomes were similar in the groups.

Almekinders et al.[3] had first described abnormal tibiofemoral positioning after an ACL injury. They suggested that untreated ACL ruptures result in irreducible anterior tibial subluxation, and this phenomenon was especially evident in patients with failed ACL reconstruction on plain radiographs. McDonald et al.[20] investigated tibiofemoral subluxation after ACL tears in more detail using magnetic resonance imaging. In their study, patients were divided into four experimental cohorts according to their ACL status: intact ACL, acute ACL disruption (within 2 months of an ACL tear), chronic ACL disruption (more than 12 months after an ACL tear), and failed ACL reconstruction. The study demonstrated significantly increased medial and lateral compartment subluxation in patients with chronic ACL disruption than in those with normal knees; however, patients with acute ACL tears did not show significant subluxation. In the current study, the mean time from injury to surgery was 9.2 and 7.2 weeks in the non-draw and draw groups, respectively. Therefore, in our study, it was too early for the tibiofemoral subluxation to have occurred. We think there would be no significant postoperative STSD difference between the two groups because subluxation did not occur. To identify the efficacy of posterior draw force during $A C L$ graft fixation in patients with tibiofemoral subluxation, further investigations with a revised $A C L$ reconstruction cohort or chronic $A C L$ deficiency cohort is necessary.

Mae et al.[19] had conducted a cadaveric investigation with respect to graft tension during ACL reconstruction. They suggested that the tibia moved proximally and posteriorly during tensioning in the graft fixation stage. After graft fixation, the proximal and posterior tibial forces caused the tibia to move proximally and posteriorly. We believe that our results support the hypothesis of the previous study. Adequate graft tensioning causes the tibia to move posteriorly; therefore, intentional posterior draw is not necessary during $A C L$ graft fixation.

This study has some limitations. First, factors, such as fixation method, graft selection, and tunnel position, could be confounding factors for postoperative stability. However, two fellowship-trained surgeons performed the same surgical procedure, except for the posterior draw force during graft fixation. Moreover, demographic data and preoperative data were similar between both groups; therefore, it was sufficient to determine the effect of posterior draw on postoperative stability after ACL reconstruction. Second, follow-up was relatively short and, therefore, survival analysis or long-term results could not be 
fully evaluated. Third, this was a retrospective study, which has inherent limitations and biases. Fourth, the posterior draw force was applied manually. Therefore, a constant force would not have been applied to the patients in the draw group, which could be a bias in interpreting the results.

\section{Conclusion}

Graft fixation with a posterior draw is not helpful in enhancing the postoperative stability during ACL reconstruction. Postoperative clinical outcomes were also not superior in the draw group.

\section{Declarations}

\section{Conflict of interest:}

The authors declare that they have no conflicts of interest.

\section{Funding:}

No funding was received for this study.

\section{Ethical approval:}

The protocol used to evaluate the radiographic findings and intraoperative navigation data was approved by our institutional review board (ISPAIK 2021-09-019).

\section{References}

1. Abebe ES, Moorman CT 3, Dziedzic TS et al (2009) Femoral tunnel placement during anterior cruciate ligament reconstruction: an in vivo imaging analysis comparing transtibial and 2-incision tibial tunnel-independent techniques. Am J Sports Med 37(10):1904-1911

2. Almekinders LC, Chiavetta JB (2001) Tibial subluxation in anterior cruciate ligament-deficient knees: Implications for tibial tunnel placement. Arthroscopy 17(9):960-962

3. Almekinders LC, Chiavetta JB, Clarke JP (1998) Radiographic evaluation of anterior cruciate ligament graft failure with special reference to tibial tunnel placement. Arthroscopy 14(2):206-211

4. Almekinders LC, Pandarinath R, Rahusen FT (2004) Knee stability following anterior cruciate ligament rupture and surgery. The contribution of irreducible tibial subluxation. J Bone Joint Surg Am 86(5):983-987

5. Anderson AF, Irrgang JJ, Kocher MS, Mann BJ, Harrast JJ, International Knee Documentation C (2006) The International Knee Documentation Committee Subjective Knee Evaluation Form: normative data. Am J Sports Med 34(1):128-135 
6. Asagumo H, Kimura M, Kobayashi Y, Taki M, Takagishi K (2007) Anatomic reconstruction of the anterior cruciate ligament using double-bundle hamstring tendons: surgical techniques, clinical outcomes, and complications. Arthroscopy 23(6):602-609

7. Balazs GC, Brelin AM, Grimm PD, Dickens JF, Keblish DJ, Rue JH (2016) Hybrid Tibia Fixation of Soft Tissue Grafts in Anterior Cruciate Ligament Reconstruction: A Systematic Review. Am J Sports Med 44(10):2724-2732

8. Bowers AL, Bedi A, Lipman JD et al (2011) Comparison of anterior cruciate ligament tunnel position and graft obliquity with transtibial and anteromedial portal femoral tunnel reaming techniques using high-resolution magnetic resonance imaging. Arthroscopy 27(11):1511-1522

9. Browning WM 3, Kluczynski MA, Curatolo C, Marzo JM (2017) Suspensory Versus Aperture Fixation of a Quadrupled Hamstring Tendon Autograft in Anterior Cruciate Ligament Reconstruction: A Metaanalysis. Am J Sports Med 45(10):2418-2427

10. Clement ND, Bardgett M, Weir D, Holland J, Gerrand C, Deehan DJ (2018) What is the Minimum Clinically Important Difference for the WOMAC Index After TKA? Clin Orthop Relat Res 476(10):2005-2014

11. Fu FH, Shen W, Starman JS, Okeke N, Irrgang JJ (2008) Primary anatomic double-bundle anterior cruciate ligament reconstruction: a preliminary 2-year prospective study. Am J Sports Med 36(7):1263-1274

12. Hulet C, Sonnery-Cottet B, Stevenson C et al (2019) The use of allograft tendons in primary ACL reconstruction. Knee Surg Sports Traumatol Arthrosc 27(6):1754-1770

13. Jin J, Yu L, Wei M, Shang Y, Wang X (2019) Comparison of efficacy and safety of different fixation devices for anterior cruciate ligament reconstruction: A Bayesian network meta-analysis protocol. Med (Baltim) 98(12):e14911

14. Kim JG, Chang MH, Lim HC et al (2015) An in Vivo 3D Computed Tomographic Analysis of Femoral Tunnel Geometry and Aperture Morphology Between Rigid and Flexible Systems in Double-Bundle Anterior Cruciate Ligament Reconstruction Using the Transportal Technique. Arthroscopy 31(7):1318-1329

15. Kim JG, Kang SH, Kim JH, Lim CO, Wang JH (2018) Comparison of Clinical Results, Second-Look Arthroscopic Findings, and MRI Findings Between the Transportal and Outside-In Techniques for Double-Bundle Anatomic Anterior Cruciate Ligament Reconstruction: A Prospective, Randomized Controlled Trial With a Minimum 2-Year Follow-up. Am J Sports Med 46(3):544-556

16. Kim JH, Oh E, Yoon YC, Lee DK, Lee SS, Wang JH (2021) Remnant-Tensioning Single-Bundle Anterior Cruciate Ligament Reconstruction Provides Comparable Stability to and Better Graft Vascularity Than Double-Bundle Anterior Cruciate Ligament Reconstruction in Acute or Subacute Injury: A Prospective Randomized Controlled Study Using Dynamic Contrast-Enhanced Magnetic Resonance Imaging. Arthroscopy 37(1):209-221

17. Kopf S, Forsythe B, Wong AK et al (2010) Nonanatomic tunnel position in traditional transtibial single-bundle anterior cruciate ligament reconstruction evaluated by three-dimensional computed 
tomography. J Bone Joint Surg Am 92(6):1427-1431

18. Lysholm J, Gillquist J (1982) Evaluation of knee ligament surgery results with special emphasis on use of a scoring scale. Am J Sports Med 10(3):150-154

19. Mae T, Shino K, Yoneda $\mathrm{K}$ et al (2020) Residual graft tension after graft fixation in anterior cruciate ligament reconstruction: Manual vs tensioning boot techniques. J Orthop Sci 25(6):1061-1066

20. McDonald LS, van der List JP, Jones KJ et al (2017) Passive Anterior Tibial Subluxation in the Setting of Anterior Cruciate Ligament Injuries: A Comparative Analysis of Ligament-Deficient States. Am J Sports Med 45(7):1537-1546

21. Muneta $\mathrm{T}$, Koga $\mathrm{H}$, Mochizuki $\mathrm{T}$ et al (2007) A prospective randomized study of 4-strand semitendinosus tendon anterior cruciate ligament reconstruction comparing single-bundle and double-bundle techniques. Arthroscopy 23(6):618-628

22. Ryu DJ, Kwon KB, Jung EY et al (2021) Clinically Reliable Knee Flexion Angle Measured on Stress Radiography for Quantifying Posterior Instability in Posterior Cruciate Ligament Injury. Orthop J Sports Med 9(3):2325967121989252

23. Sanders JO, Brown GA, Murray J, Pezold R, Sevarino KS (2016) Treatment of Anterior Cruciate Ligament Injuries. J Am Acad Orthop Surg 24(8):e81-83

24. Sanders TL, Maradit Kremers H, Bryan AJ et al (2016) Incidence of Anterior Cruciate Ligament Tears and Reconstruction: A 21-Year Population-Based Study. Am J Sports Med 44(6):1502-1507

25. Tanaka MJ, Jones KJ, Gargiulo AM et al (2013) Passive anterior tibial subluxation in anterior cruciate ligament-deficient knees. Am J Sports Med 41(10):2347-2352

26. Yoshiya S, Kurosaka M, Ouchi K, Kuroda R, Mizuno K (2002) Graft tension and knee stability after anterior cruciate ligament reconstruction.Clin Orthop Relat Res(394):154-160

\section{Figures}




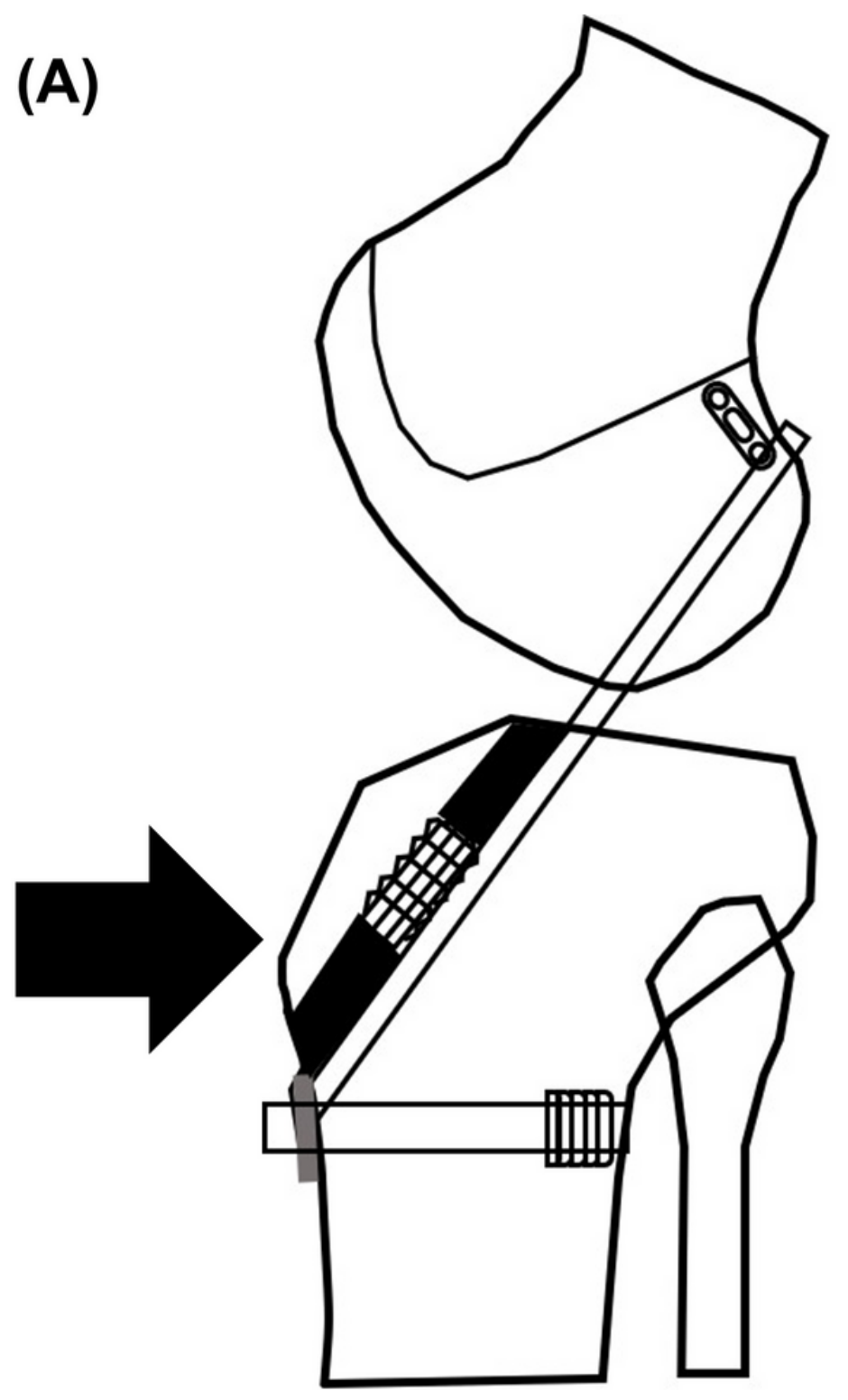

(B)

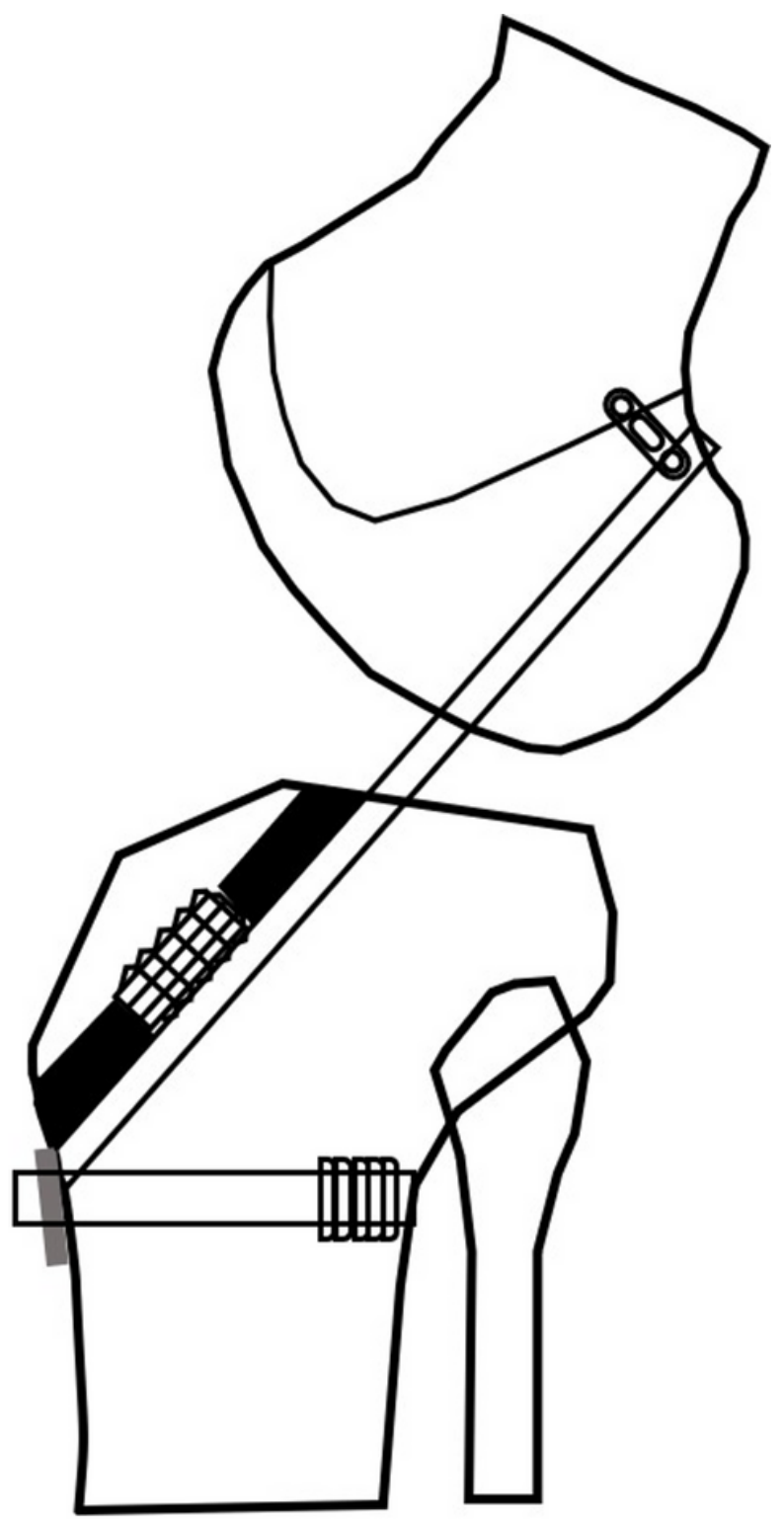

Figure 1

Schematic view of (A) the posterior draw force applied or (B) posterior draw force not applied during graft fixation of anterior cruciate ligament reconstruction 


\section{Figure 2}

Intraoperative view of (A) posterior draw force applied or (B) posterior draw force not applied during anterior cruciate ligament reconstruction 


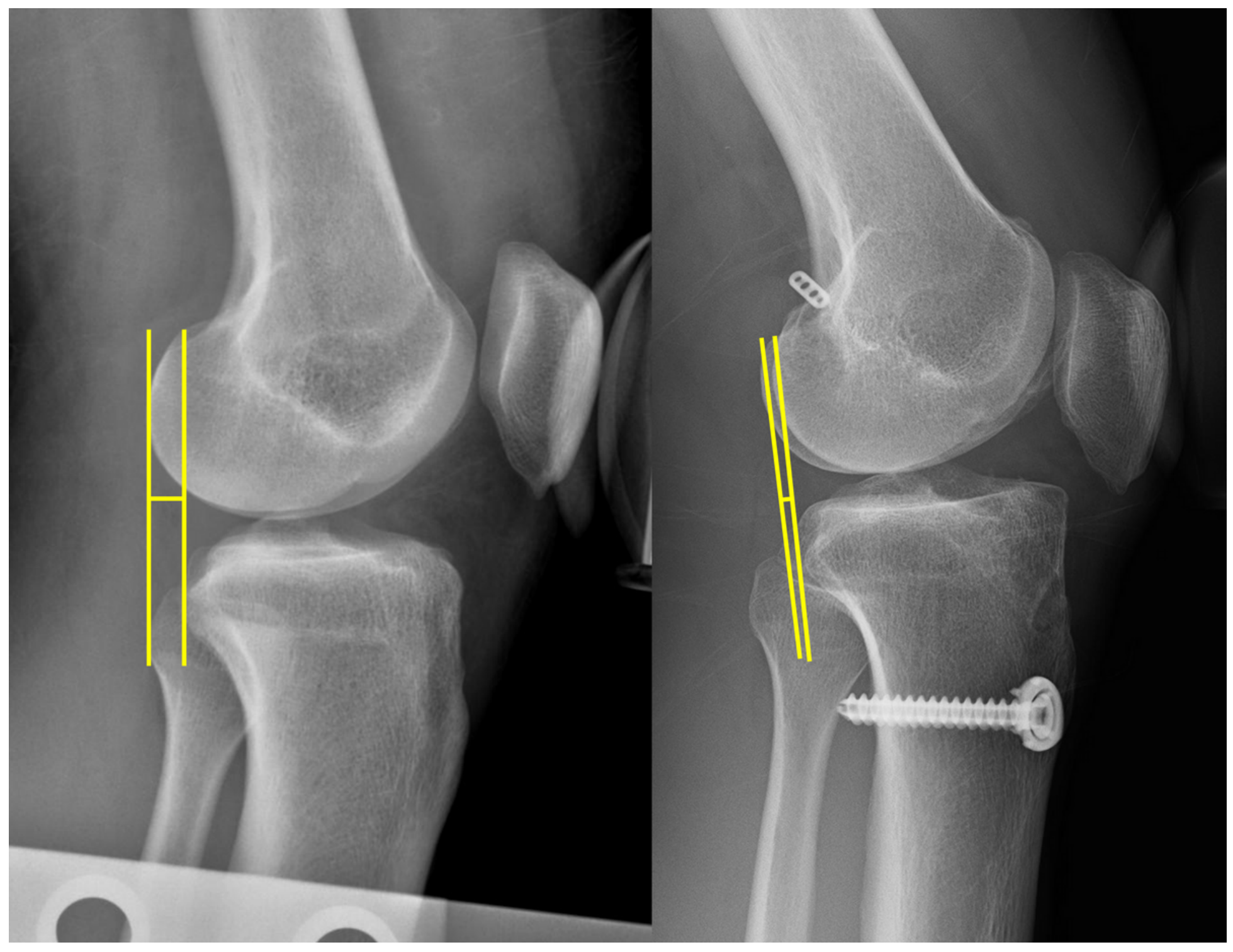

\section{Figure 3}

Measurement of preoperative and postoperative anterior tibial displacement on anterior stress radiograph. Side-to-side difference (STSD) was defined as the difference of anterior tibial displacement between the injured and contralateral knees. 